Pacific Journal of Mathematics

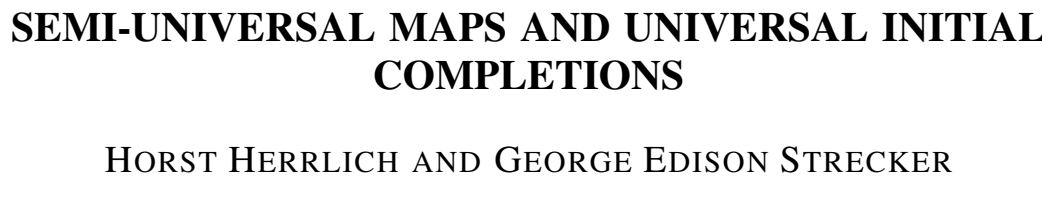




\title{
SEMI-UNIVERSAL MAPS AND UNIVERSAL INITIAL COMPLETIONS
}

\author{
H. Herrlich and G. E. Strecker
}

Initial completions of categories $(A, U)$ over a base category are investigated simultaneously with certain generalizations of the notion of topological functor. The main result states that $(\underline{A}, U)$ has a reflective universal initial completion if and only if the functor $U$ is topologically algebraic in the sense of $Y$. H. Hong. This is analogous to results of Hoffmann, Tholen, and Wischnewsky that $(\underline{A}, U)$ has a reflective Mac Neille completion if and only if $U$ is semi-topological. In addition, the class of sem-itopological functors is shown to be the smallest class closed under composition and containing all topologically algebraic functors. It is also shown that for any $(\underline{E}, \underline{M})$-functor $U$ (resp. $(E, \underline{M})$-category) $\underline{E}$ must be contained in the class of generating $U$-morphisms (resp. epimorphisms). Specific constructions of the above completions are given, the first necessitating the new concept of semi-universal morphism. Examples illuminating the theory are also provided.

\section{o. Background.}

0.1. Let $X$ be a category. We investigate categories over $X$, i.e., pairs $(\underline{A}, U)$ where $\underline{A}$ is a category and $U: \underline{A} \rightarrow \underline{X}$ is a faithful, amnestic functor, and functors $F:(\underline{A}, U) \rightarrow(\underline{B}, V)$ over $\underline{X}$; i.e., functors $F: \underline{A} \rightarrow \underline{B}$ with $V \cdot F=U$. As has been shown in [6], every small category $(\underline{A}, U)$ over $\underline{X}$ has several distinguished initial completions, in particular:

(a) A Mac Neille completion $E^{4}:(\underline{A}, U) \rightarrow\left(\underline{A}^{4}, U^{4}\right)$, which is characterized either as the smallest initial completion of $(\underline{A}, U)$, or as the initial completion which is both initially and finally dense, or as the injective hull of $(\underline{A}, U)$ in the category of small categories over $\underline{X}$ and functors over $\underline{X}$.

(b) A universal initial completion $E^{3}:(\underline{A}, U) \rightarrow\left(\underline{A}^{3}, U^{3}\right)$, which is characterized either as the largest initiality preserving, initially dense, full extension of $(\underline{A}, U)$, or as the initially complete reflection of $(\underline{A}, U)$ in the category of small categories over $\underline{X}$ and initiality preserving functors over $\underline{X}$.

If $\underline{A}$ is large, initial completions of $(\underline{A}, U)$ need not exist, even for small $\underline{X}$. For instance, if $\underline{X}$ is the terminal category, i.e., the category with precisely one morphism, then (initially complete) cate- 
gories over $\underline{X}$ are essentially the same as (complete) partially ordered classes, and

(a) The partially ordered class $(\Omega \times\{0,1\}$, $\leqq$, with $\Omega$ being a proper class and

$$
(\omega, n)<\left(\omega^{\prime}, n^{\prime}\right) \Longleftrightarrow\left(n<n^{\prime} \text { and } \omega \neq \omega^{\prime}\right),
$$

has no (initial) completion, equivalently no Mac Neille completion.

(b) The partially ordered class $(\Omega, \leqq)$, with $\Omega$ being a proper class and

$$
\omega \leqq \omega^{\prime} \Longleftarrow \omega=\omega^{\prime}
$$

has a Mac Neille completion but no universal initial completion.

The problem of characterizing those categories $(\underline{A}, U)$ over $\underline{X}$, which have specific initial completions, has been of considerable interest. In case the problem is restricted to small-fibered categories, i.e., if the (specific) completions are required to be $S$-categories in the sense of Hušek [16], resp. top-categories in the sense of Wyler $[29,30]$, it has been solved for the Mac Neille case and $\underline{X}=$ Set by Kučera and Pultr [17]. Recently it has been solved in full generality and in its small-fiberd version by Adámek, Herrlich and Strecker [2].

0.2. Surprisingly new light is thrown on the above problems by recent investigations of a different kind. The concept of topological functors has been subject to various generalizations. The following concepts are particularly interesting:

(a) $(\underline{E}, \underline{M})$-topological functors (Herrlich [4], Hoffmann [8], Marny [18], Brümmer [3], Wolff [27, 28]).

(b) Topologically-algebraic functors (Y. H. Hong [15], S. S. Hong [14]).

(c) Orthogonal $\underline{M}$-functors (Tholen $[19,20,21]$, Wischnewsky [26]).

(d) Orthogonal $Q$-functors (Tholen [23]).

(e) Semi-topological functors (Trnkova, [25] Hoffmann [9, 11, 12 , 13], Tholen [22, 23, 24], Wischnewsky [24, 26]).

It is known that (a) is strictly stronger than the other 4 concepts, (c) and (d) are equivalent (Tholen [23]) and each of the above concepts implies (e). Answering the remaining open questions, concerning the relationships between the above concepts, we will show that:

(1) The concepts (b), (c) and (d) are equivalent (Theorem 2.3 below); 
(2) The concept (b) is strictly stronger than (e), but agrees with (e) under fairly mild side conditions (Herrlich, Nakagawa, Strecker, Titcomb [7]).

(3) The class of semi-topological functors is the smallest class of functors containing all topologically-algebraic functors and being closed under composition (Theorem 2.8 below).

A crucial idea, leading to the above result (1), is the observation-highly interesting in itself-that for any $(\underline{E}, \underline{M})$-functor, the class $\underline{E}$ must consist of generating $U$-morphisms only (Theorem 2.1 below).

0.3. Initial completions and generalized topological functors are linked by the following concept: an initial completion $(\underline{A}, U) \hookrightarrow(\underline{B}, V)$ is called (epi) reflective, provided the embedding $\underline{A} \hookrightarrow \underline{B}$ has a left-adjoint (with epimorphic front adjunctions)-which need not be a functor over $\underline{X}$. The following hold:

(1) $(\underline{A}, U)$ has an epireflective Mac Neille completion iff $U$ is (epi, $\underline{M}$ )-topological, provided the base category $\underline{X}$ is an (epi, $\underline{M}$ )category (Herrlich [4]).

(2) $(\underline{A}, U)$ has a reflective Mac Neille completion iff $U$ is semitopological (Hoffmann [13], Tholen and Wischnewsky [24, 26]).

(3) $(\underline{A}, U)$ has a reflective universal initial completion iff $U$ is topologically-algebraic (Theorem 2.7 below).

The basic idea, providing insight in the above results, is the following. Initial completions, with specific properties as mentioned above, exist, even for large categories $\underline{A}$, in some higher universe (cf. the constructions in [6]). The objects of the larger "categories" are just suitable $U$-sources $\left(X \stackrel{f_{i}}{\rightarrow} U A_{i}\right)_{I}$. Since such sources usually will be proper classes, they cannot be objects of categories in the given universe. The basic idea to construct reflective initial completions of a certain kind is to represent any $U$-source $\left(X \stackrel{f_{i}}{\rightarrow} U A_{i}\right)_{I}$ of the corresponding type by a single generating $U$-morphism $X \stackrel{g}{\rightarrow} U A$ such that the following holds: a $U$-morphism $X \stackrel{f}{\rightarrow} U B$ belongs to the given $U$-source iff there exists an $\underline{A}$-morphism $h: A \rightarrow B$ with $f=$ $(U h) \cdot g$. In case this is possible, an initial completion of the desired kind can be constructed in the given universe, by taking as objects just those $U$-morphisms $X \stackrel{g}{\rightarrow} U A$, which represent some $U$-source $\left(X \stackrel{f_{i}}{\rightarrow} U A_{i}\right)_{I}$ of the corresponding type. In the case of

(1) reflective Mac Neille completions, they are characterized 
as semi-final $U$-morphisms;

(2) reflective universal initial completions, they are characterized as semi-universal $U$-morphisms, a crucial concept introduced below (1.4).

1. Basic tools. Throughout this paper, $U: \underline{A} \rightarrow \underline{X}$ will be assumed to be a faithful and amnestic ${ }^{1}$ functor. (Neither restriction is serious. In fact all of our results not concerning initial completions hold without either restriction.)

1.1. A $U$-morphism with domain $X$ and codomain $A$ is a pair $(f, A)$, where $A$ is an $\underline{A}$-object and $f: X \rightarrow U A$ is an $X$-morphism with domain $X$ and codomain $U A$. We will usually denote such a $U$-morphism suggestively by $X \stackrel{f}{\rightarrow} U A$. If $U A \stackrel{g}{\rightarrow} U B$ is an $X$-morphism, for which there is a (unique) $\underline{A}$-morphism $\bar{g}: A \rightarrow B$ with $U \bar{g}=g$, we will sometimes express this by writing (inaccurately but suggestively) that $U A \stackrel{g}{\rightarrow} U B$ is an $\underline{A}$-morphism (thus not distinguishing between $g$ and $\bar{g}$ ).

A $U$-morphism $X \stackrel{f}{\rightarrow} U A$ will be said to generate $A$ provided that for each $\underline{A}$-object $\hat{A}$ and each pair of $A$-morphisms $r, s: A \rightarrow \hat{A}$ the equality $U r \cdot f=U s \cdot f$ implies $r=s$.

1.2. A $U$-source $\mathscr{S}$ with domain $X$ and codomain $\left(A_{i}\right)_{I}$ is a pair $(X, \xi)$ where $X$ is an $\underline{X}$-object and $\xi$ is a family of $U$-morphisms $X \stackrel{f_{i}}{\rightarrow} U A_{i}$ indexed by a class $I$. Such a $U$-source will usually be denoted by $\left(X \stackrel{f_{i}}{\rightarrow} U A_{i}\right)_{I}$. (Notice that, if $U$ is the identity functor, then a $U$-morphism is essentially just an $\underline{A}$-morphism and a $U$-source is essentially an $\underline{A}$-source in the usual sense.) A $U$-morphism is said to belong to $\mathscr{S}$ if it is of the form $\left(f_{i}, A_{i}\right)$ for some $i \in I$.

If $\mathscr{S}=\left(X \stackrel{f_{i}}{\rightarrow} U A_{i}\right)_{I}$ is a $U$-source, $X \stackrel{e}{\rightarrow} U A$ is a $U$-morphism and $\left(A \stackrel{m_{i}}{\rightarrow} A_{i}\right)_{I}$ is an $\underline{A}$-source such that for each $i \in I$

$$
X \stackrel{e}{\longrightarrow} U A \stackrel{U m_{i}}{\longrightarrow} U A_{i}=X \stackrel{f_{i}}{\longrightarrow} U A_{i}
$$

then this will be called a $U$-factorization of $\mathscr{S}$. If $\underline{E}$ is a class of $U$-morphisms and $M$ is a collection of $\underline{A}$-sources, such that $(e, A)$ belongs to $\underline{E}$ and $\left(A \stackrel{m_{i}}{\rightarrow} A_{i}\right)_{I}$ belongs to $\underline{M}$, the above factorization will be called an $(\underline{E}, \boldsymbol{M})$-factorization of $\mathscr{S}$. If every $U$-source has some $(\underline{E}, \underline{M})$-factorization, the functor $U$ will be called $(\underline{E}, \underline{M})$-factorizable. If morever for any $X \stackrel{e}{\rightarrow} U A$ in $\underline{E}$, any $\left(A^{\prime} \stackrel{m_{i}}{\rightarrow} A_{\imath}\right)_{I}$ in $\underline{M}$,

$1 U$ is called amnestic, provided any $\underline{A}$-isomorphism $f$, with $U f$ being an $\underline{X}$-identity, must be an $\underline{A}$-identity. 
any $U$-morphism $X \stackrel{f}{\rightarrow} U A^{\prime}$, and any $\underline{A}$-source $\left(A \stackrel{f_{2}}{\rightarrow} A_{i}\right)_{I}$ with $\left(U f_{i}\right)$. $e=\left(U m_{i}\right) \cdot f$ for each $i \in I$, there exists a unique $\underline{A}$-morphism $g: A \rightarrow$ $A^{\prime}$ such that the diagram

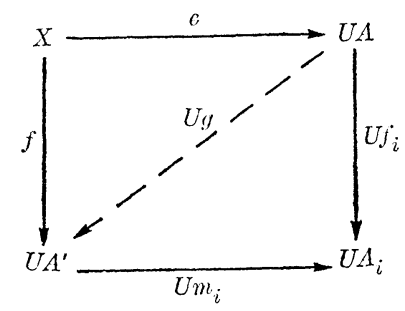

commutes for each $i \in I$, the functor $U$ will be called an $(\underline{E}, \underline{M})$ functor. If, for a given class $\underline{E}$ of $U$-morphisms, there exists some $\underline{M}$ such that $U$ is an $(\underline{E}, \underline{M})$-functor, we will call $U$ an $(\underline{E},-)$ functor. Similarly, (E, -)-factorizations, (-, $\underline{M})$-functors, etc. If the identity functor on $\underline{A}$ is an $(\underline{E}, \underline{M})$-functor then $\underline{A}$ is called an $(\underline{E}, \underline{M})$-category.

An $\underline{A}$-source $\left(A \stackrel{m_{i}}{\rightarrow} A_{i}\right)_{K}$ is called $U$-initial, or just initial, provided an $\underline{X}$-morphism $U A^{\prime} \stackrel{h}{\rightarrow} U A$ is an $\underline{A}$-morphism if all $U A^{\prime} \stackrel{\left(U m_{i}\right) \cdot h}{\rightarrow} U A_{i}$ are $\underline{A}$-morphisms. The dual concept is called (U)-final sink.

1.3. A functor $U: A \rightarrow \underline{X}$ is called:

- topological, provided it is (identity, initial)-factorizable;

- topologically-algebraic, provided it is (generating, initial)factorizable;

- semi-topological, provided for each $U$-sink $\left(U A_{i} \stackrel{f_{i}}{\rightarrow} X\right)_{I}$ there exists an $\underline{A}$-sink $\left(A_{i} \stackrel{g_{i}}{\rightarrow} A\right)_{I}$ and a $U$-morphism $X \stackrel{e}{\rightarrow} U A$ with $U g_{i}=$ $e \cdot f_{i}$ for each $i \in I$ and such that for any $\underline{A}-\operatorname{sink}\left(A_{i} \stackrel{\tilde{g}_{i}}{\rightarrow} \widetilde{A}\right)_{I}$ and any $U$-morphism $X \stackrel{\widetilde{e}}{\rightarrow} U \widetilde{A}$ with $U \widetilde{g}_{i}=\widetilde{e} \cdot f_{i}$ for each $i \in I$ there exists a unique $\underline{A}$-morphism $g: A \rightarrow \widetilde{A}$ with $(U g) \cdot e=\widetilde{e}$, i.e., such that the following diagram commutes;

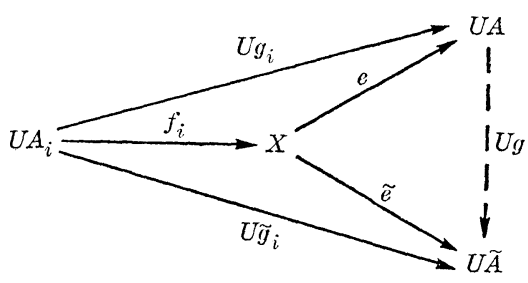

$U$-morphisms $X \stackrel{e}{\rightarrow} U A$, appearing in the above setting, are called 
semi-final solutions of the $U$-sink $\left(U A_{i} \stackrel{f_{i}}{\rightarrow} X\right)_{I}$, resp. semi-final $U$ morphisms.

Obviously, every topological functor is topologically-algebraic, and every topologically-algebraic functor is semi-topological. Moreover, every full reflective embedding, every regular functor [5], and every monadic functor into $S e t$ is topologically-algebraic. But, in general (even for the base category of relations), monadic functors may fail to be semi-topological (Adámek [1]). The following two concepts will be crucial tools for our investigations.

1.4. A $U$-morphism $X \stackrel{e}{\rightarrow} U A$ is called semi-universal, provided for any initial source $\left(A^{\prime} \stackrel{m_{i}}{\rightarrow} A_{i}\right)_{I}$, any $\underline{A}$-source $\left(A \stackrel{f_{i}}{\rightarrow} A_{i}\right)_{I}$, and any $U$-morphism $X \stackrel{f}{\rightarrow} U A^{\prime}$ with $\left(U m_{i}\right) \cdot f=\left(U f_{i}\right) \cdot e$ there exists a unique $A$-morphism $g: A \rightarrow A^{\prime}$ such that the above diagram (*) commutes for each $i \in I$.

Proposition 1.5. (a) Every universal U-morphism is semifinal.

(b) Every semi-final U-morphism is semi-universal.

(c) Every semi-universal U-morphism is generating.

Proof. (a) Every universal $U$-morphism $X \stackrel{e}{\rightarrow} U A$ is a semifinal solution of the $\operatorname{sink}(\phi, X)$.

(b) Let $X \stackrel{e}{\rightarrow} U A$ be a semi-final solution of $\left(U B_{k} \stackrel{g_{k}}{\rightarrow} X\right)_{K}$. If $\left(A^{\prime} \stackrel{m_{i}}{\rightarrow} A_{i}\right)_{I}$ is an initial source, $X \stackrel{f}{\rightarrow} U A^{\prime}$ is a $U$-morphism, and $\left(A \stackrel{f_{i}}{\rightarrow} A_{i}\right)_{I}$ is a source in $\underline{A}$ with $\left(U m_{i}\right) \cdot f=\left(U f_{i}\right) \cdot e$ for each $i \in I$, then-by initiality-each $U B_{K} \stackrel{f \cdot g_{k}}{\longrightarrow} U A^{\prime}$ is an $\underline{A}$-morphism. Hence there exists a unique $\underline{A}$-morphism $g: A \rightarrow A^{\prime}$ with $f=(U g) \cdot e$, i.e., such that $(*)$ commutes.

(c) Let $X \stackrel{e}{\rightarrow} U A$ be semi-universal, and let $f_{i}: A \rightarrow A^{\prime}$ be $\underline{A}$ morphisms with $\left(U f_{1}\right) \cdot e=\left(U f_{2}\right) \cdot e=f$. For $I=\{1,2\}, m_{i}=1_{A^{\prime}}$ and $A_{i}=A^{\prime}$, the source $\left(A^{\prime} \stackrel{m_{i}}{\rightarrow} A_{i}\right)_{I}$ is initial. Hence there exists a unique $\underline{A}$-morphism $g: A \rightarrow A^{\prime}$ such that the diagram (*) commutes. Hence $f_{1}=g=f_{2}$.

1.6. The standard enrichment of a $U$-source $\mathscr{S}=\left(X \stackrel{f_{i}}{\rightarrow} U A_{i}\right)_{I}$ is the smallest $U$-source $\hat{\mathscr{S}}=\left(X \stackrel{f_{j}}{\rightarrow} U A_{j}\right)_{J}$ having the following properties:

(a) $I \subseteq J$ and the family $\left(f_{i}, A_{i}\right)_{I}$ is the corresponding restriction of the family $\left(f_{j}, A_{j}\right)_{J}$.

(b) If $X \stackrel{e}{\rightarrow} U A$ belongs to $\hat{\mathscr{S}}$ and $A \stackrel{h}{\rightarrow} \bar{A}$ is any $\underline{A}$-morphism, then $X \stackrel{e}{\rightarrow} U A \stackrel{U h}{\longrightarrow} U \bar{A}$ belongs to $\hat{\mathscr{S}}$. 
(c) If $X \stackrel{e}{\rightarrow} U A$ is a $U$-morphism and $\left(A \stackrel{m_{k}}{\rightarrow} A_{k}\right)_{K}$ is an initial $\underline{A}$ source with each $X \stackrel{e}{\rightarrow} U A \stackrel{U m_{k}}{\longrightarrow} U A_{k}$ belonging of $\hat{\mathscr{S}}$, then $X \stackrel{e}{\rightarrow} U A$ belongs to $\hat{\mathscr{S}}$.

Proposition 1.7. Let $\mathscr{S}=\left(X \stackrel{f_{i}}{\rightarrow} U A_{i}\right)_{I}$ be a U-source, $\hat{\mathscr{S}}=$ $\left(X \stackrel{f_{j}}{\rightarrow} U A_{j}\right)_{J}$ be its standard enrichment, and

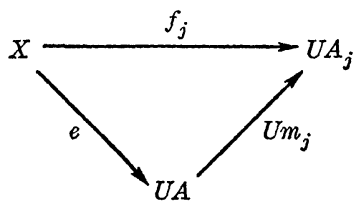

be some (generating, - )-factorization of $\hat{\mathscr{S}}$. Then conditions (1), (2), and (3) below are equivalent and imply (4):
(1) $\left(A \stackrel{m_{i}}{\rightarrow} A_{i}\right)_{I}$ is initial.
(2) $\left(A \stackrel{m_{j}}{\rightarrow} A_{j}\right)_{J}$ is initial.
(3) $X \stackrel{e}{\rightarrow} U A$ belongs to $\hat{\mathscr{S}}$.
(4) $X \stackrel{e}{\rightarrow} U A$ is semi-universal.

Proof. Clearly (1) implies (2), and (2) implies (3).

$(3) \Rightarrow(4)$. Let $\left(A^{\prime} \stackrel{n_{k}}{\rightarrow} A_{k}^{\prime}\right)_{K}$ be an initial source, $\left(A \stackrel{g_{k}}{\rightarrow} A_{k}^{\prime}\right)_{K}$ be an A-source, and $X \stackrel{f}{\rightarrow} U A^{\prime}$ be a $U$-morphism with $\left(U n_{k}\right) \cdot f=\left(U g_{k}\right) \cdot e$ for each $k \in K$. By $1.6(\mathrm{~b})$ every $X \stackrel{e}{\rightarrow} U A \stackrel{U g_{k}}{\longrightarrow} U A_{k}^{\prime}$ belongs to $\hat{\mathscr{S}}$. So by 1.6 (c) $X \stackrel{f}{\rightarrow} U A^{\prime}$ belongs to $\hat{\mathscr{S}}$. Consequently, these exists some $j \in J$ with $\left(f, A^{\prime}\right)=\left(f_{j}, A_{j}\right)$. Since $X \stackrel{e}{\rightarrow} U A$ generates $A$, this implies that $g=m_{j}$ is the unique $\underline{A}$-morphism making the following diagram commute:

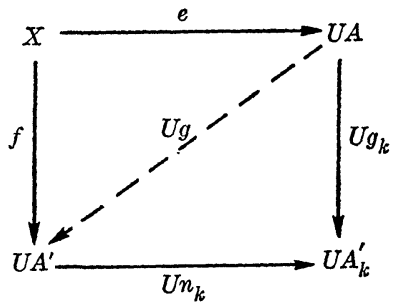

$(3) \Rightarrow(2)$. If $(e, A)=\left(f_{j}, A_{j}\right)$, then $m_{j}=1_{A_{j}}$, which implies that $\left(A \stackrel{m_{j}}{\rightarrow} A_{j}\right)_{J}$ is initial.

$(2) \Rightarrow(1)$. Let $\left(A \stackrel{m_{j}}{\rightarrow} A_{j}\right)_{J}$ be initial. Let $U B \stackrel{h}{\rightarrow} U A$ be an $\underline{X}$-mor- 
phism, such that $U B \stackrel{\left(U m_{2}\right) \cdot h}{\rightarrow} U A_{i}$ is an $A$-morphism for each $i \in I$. Consider the subclass $K$ of $J$ consisting of those $k \in J$, for which $U B \stackrel{\left(U m_{k}\right) \cdot h}{\rightarrow} U A_{k}$ is an $\underline{A}$-morphism. It is easily seen that the $U$ source $\left(X \stackrel{f_{k}}{\rightarrow} U A_{k}\right)_{K}$ satisfies all 3 conditions in 1.6 , and therefore $K$ must equal $J$. Initiality of $\left(A \stackrel{m_{j}}{\rightarrow} A_{j}\right)_{J}$ implies that $U B \stackrel{h}{\rightarrow} U A$ is an A-morphism.

\section{Main results.}

THEOREM 2.1. If $U: \underline{A} \rightarrow \underline{X}$ is an (E, $\underline{M})$-functor then each member of $\underline{E}$ must be a generating U-morphism.

Proof. Let $U: \underline{A} \rightarrow \underline{X}$ be an $(\underline{E}, \underline{M})$-functor. Assume that $\underline{E}$ contains some nongenerating $U$-morphism $X \stackrel{e}{\rightarrow} U A$. Then there would exist distinct $A$-morphisms $r, s: A \rightarrow \hat{A}$ with $U r \cdot e=U s \cdot e=k$. Let $I$ be the class of all $A$-morphisms, and, for each $i \in I$, let $X \stackrel{f_{i}}{\rightarrow} U A_{i}$ be $X \stackrel{k}{\rightarrow} U \hat{A}$. Then the $U$-source $\left(X \stackrel{f_{i}}{\rightarrow} U A_{i}\right)_{I}$ would have an $(\underline{E}, \underline{M})$ factorization $X \stackrel{f_{i}}{\rightarrow} U A_{i}=X \stackrel{e^{\prime}}{\rightarrow} U A^{\prime} \stackrel{U m_{i}}{\longrightarrow} U A_{i}$. For each $f \in I$ define:

$$
g_{f}=\left\{\begin{array}{l}
r \text { if } m_{f} \cdot f=s \\
s \text { otherwise }
\end{array}\right.
$$

Then, for each $i \in I, U g_{i} \cdot e=k=U m_{i} \cdot e^{\prime}$ so that by the diagonal property there would be a unique $h: A \rightarrow A^{\prime}$ such that for each $i \in I$ the diagram

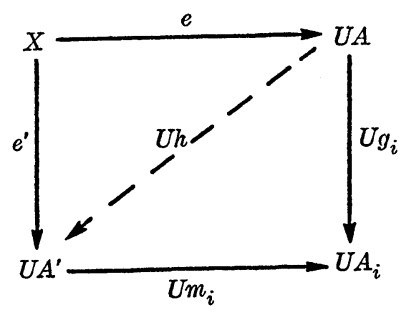

commutes. In particular

$$
m_{h} \cdot h=g_{h}=\left\{\begin{array}{l}
r \text { if } m_{h} \cdot h=s \\
s \text { if } m_{h} \cdot h \neq s,
\end{array}\right.
$$

which is impossible.

For a corresponding result concerning locally orthogonal $Q$-functors see Tholen [23], 6.4.

Proposition 2.2. The following are equivalent: 
(1) U has a left adjoint.

(2) $U$ is a (universal, source)-functor.

(3) $U$ is a (-, -)-functor.

(4) $U$ is (generating, -)-factorizable.

Proof. $\quad(4) \Rightarrow(1) \Longrightarrow(2) \Longrightarrow(3)$ is obvious. $\quad(3) \Rightarrow(4)$ is immediate from Theorem 2.1 .

THEOREM 2.3. The following are equivalent:

(1) $U$ is topologically-algebraic.

(2) $U$ is a (semi-universal, initial)-functor.

(3) $U$ is a $(Q,-)$-functor with every $U$-morphism that is a $\underline{X}$-isomorphism belonging to $Q$ (i.e., $U$ is an orthogonal $Q$-functor in the sense of Tholen [23]).

(4) $U$ is a (-, $\underline{M})$-functor with each source in $\underline{M}$ being initial (i.e., $U$ is an orthogonal $\underline{M}$-functor in the sense of Tholen [19, 20, 21]).

\section{Proof.}

$(1) \Rightarrow(2)$. Let $\mathscr{S}=\left(X \stackrel{f_{2}}{\rightarrow} U A_{i}\right)_{I}$ be any $U$-source, $\hat{\mathscr{S}}=\left(X \stackrel{f_{j}}{\rightarrow} U A_{j}\right)_{J}$ be its standard enrichment and $\left(X \stackrel{e}{\rightarrow} U A \stackrel{U m_{j}}{\longrightarrow} U A_{j}\right)_{J}$ be the (generating, initial)-factorization of $\hat{\mathscr{S}}$. By Proposition 1.7, $\left(A \stackrel{m_{i}}{\rightarrow} A_{i}\right)_{I}$ is initial and $X \stackrel{e}{\rightarrow} U A$ is semi-universal.

$(2) \Rightarrow(3)$. Trivial.

$(3) \Rightarrow(4)$. Let $\left(A \stackrel{m_{i}}{\rightarrow} A_{i}\right)_{I}$ be a source in $\underline{M}$ and $U \hat{A} \stackrel{h}{\rightarrow} U A$ a $U$ morphism such that, for each $i \in I, m_{i} \cdot h=U f_{i}$. Then the outer square

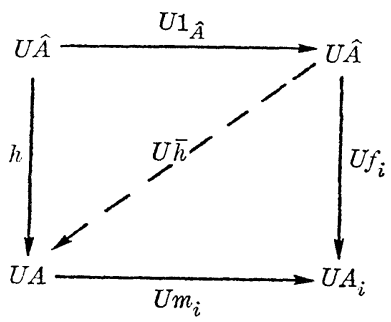

commutes. Since $\left(U 1_{\hat{A}}, \hat{A}\right)$ belongs to $\underline{E}$ there exists a unique $\underline{A}$ morphism $\bar{h}: \hat{A} \rightarrow A$ such that the above diagram commutes, which implies $h=U \bar{h}$. Hence $\left(A \stackrel{m_{i}}{\rightarrow} A_{i}\right)_{I}$ is initial.

$(4) \Rightarrow(1)$. If $U$ is an $(\underline{E}, \underline{M})$-functor with each source in $\underline{M}$ 
initial then by Theorem 2.1 each member of $\underline{E}$ is generating so that $U$ is (generating, initial)-factorizable.

The equivalence of (3) and (4) in the above theorem has been obtained previously by Tholen [23].

2.4. A category over $\underline{X}$ is a pair $(\underline{A}, U)$ where $U: \underline{A} \rightarrow \underline{X}$ is a faithful, amnestic functor. A functor over $\underline{X}$ between categories over $\underline{X},(\underline{A}, U) \stackrel{F}{\rightarrow}(\underline{B}, V)$ is a functor $F: \underline{A} \rightarrow \underline{B}$ for which $U=V \cdot F$. Such a functor over $\underline{X}$ is said to be initiality preserving provided that for each $U$-initial source $\left(A \stackrel{m_{i}}{\rightarrow} A_{i}\right)_{I}$ the source $\left(F A \stackrel{F m_{i}}{\longrightarrow} F A_{i}\right)_{I}$ is a $V$-initial source. It is called initially dense provided that each $\underline{B}$-object is the domain of some $V$-initial source with codomain in $F[\underline{A}]$. The dual concept is called finally dense. An initial completion of a category $(\underline{A}, U)$ over $X$ is a full embedding $(\underline{A}, U) \stackrel{F}{\rightarrow}(\underline{B}, V)$ over $\underline{X}$ for which $(\underline{B}, V)$ is initially complete (i.e., for which $V: \underline{B} \rightarrow \underline{X}$ is a topological functor). An initial completion $(\underline{A}, U) \stackrel{E}{\rightarrow}$ $(\underline{B}, V)$ is called reflective if $E$ has a left adjoint $R: \underline{B} \rightarrow \underline{A}$ (where $R$ need not be a functor over $\underline{X})$. An initial completion $(\underline{A}, U) \stackrel{E}{\rightarrow}$ $(\underline{B}, V)$ is called universal provided that $E$ is initiality preserving and has the property that for every initially complete category $(\underline{C}, W)$ over $X$, every initiality preserving functor $(\underline{A}, U) \stackrel{F}{\rightarrow}(\underline{C}, W)$ over $\underline{X}$ has a unique initiality preserving extension $(\underline{B}, V) \stackrel{G}{\rightarrow}(\underline{C}, W)$ over $\underline{X}$. An initial completion is called a Mac Neille completion provided that it is initially and finally dense.

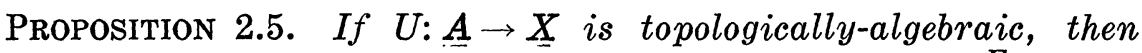
$(\underline{A}, U)$ has a reflective universal initial completion $(\underline{A}, U) \stackrel{E}{\rightarrow}(\underline{B}, V)$.

Proof. (i) Construction of $(\underline{B}, V)$ :

The objects of $\underline{B}$ are all semi-universal $U$-morphisms $X \stackrel{e}{\rightarrow} U A$. A $\underline{B}$-morphism from $X \stackrel{e}{\rightarrow} U A$ to $X^{\prime} \stackrel{e^{\prime}}{\rightarrow} U A^{\prime}$ is any pair $(f, k)$ where $X \stackrel{\stackrel{f}{\rightarrow}}{\rightarrow} X^{\prime}$ is an $\underline{X}$-morphism, $A \stackrel{k}{\rightarrow} A^{\prime}$ is an $\underline{A}$-morphism and the square

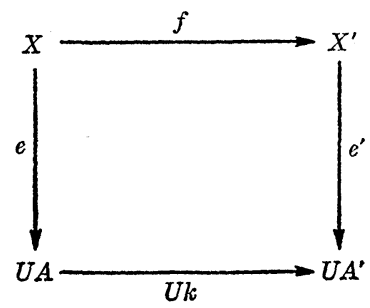


commutes. Composition is defined coordinatewise using the compositions in $\underline{X}$ and in $\underline{A}$. The faithful functor $V: \underline{B} \rightarrow \underline{X}$ is defined by

$$
V(X \stackrel{e}{\longrightarrow} U A)=X
$$

and

$$
V(f, k)=f .
$$

In general, $V$ is not amnestic, which is irrelevant here. The extension $E:(\underline{A}, U) \rightarrow(\underline{B}, V)$ is defined by

$$
E(A)=U A \stackrel{1_{U A}}{\longrightarrow} U A
$$

and

$$
E(f)=(U f, f) .
$$

(ii) $E A$ is reflective in $B$.

For any $\underline{B}$-object $B=(X \stackrel{e}{\rightarrow} U A)$, its $E \underline{A}$-reflection is given by

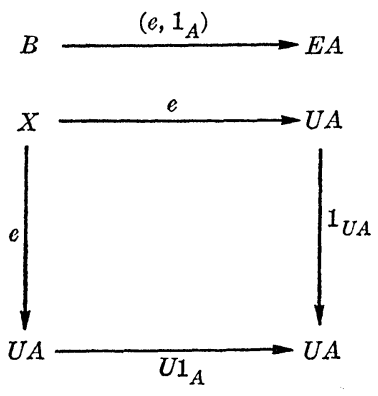

(iii) $(\underline{B}, V)$ is initially complete.

Let $\left(X \stackrel{f_{i}}{\rightarrow} V B_{i}\right)_{I}$ be a $V$-source, where $B_{i}=\left(X_{i} \stackrel{e_{i}}{\rightarrow} U A_{i}\right)$ for each $i \in I$. By Theorem 2.3 the $U$-source $\left(X \stackrel{e_{i} \cdot f_{i}}{\longrightarrow} U A_{i}\right)_{I}$ has a (semi-universal, $U$-initial)-factorization $X \stackrel{e}{\rightarrow} U A \stackrel{U m_{i}}{\longrightarrow} U A_{i}$. Put $B=(X \stackrel{e}{\rightarrow} U A)$. It remains to show that the $\underline{B}$-source $\left(B \stackrel{\left(f_{i}, m_{i}\right)}{\rightarrow} B_{i}\right)_{I}$ is $V$-initial. Let $\hat{B}=(Y \stackrel{\hat{e}}{\rightarrow} U \hat{A})$ be a $\underline{B}$-object, $h: V \hat{B} \rightarrow V B$ an $\underline{X}$-morphism, and $\left(\hat{B} \stackrel{\left(h_{i}, g_{i}\right)}{\longrightarrow} B_{i}\right)_{I}$ a $\underline{B}$-source with $V\left(h_{i}, g_{i}\right)=V\left(f_{i}, m_{i}\right) \cdot h$ for each $i \in I$. Since $Y \stackrel{\hat{e}}{\rightarrow} U \hat{A}$ is semi-universal and $\left(A \stackrel{m_{i}}{\rightarrow} A_{i}\right)_{I}$ is $U$-initial there exists a unique $\underline{A}$-morphism $g: \hat{A} \rightarrow A$ such that the following diagram commutes: 


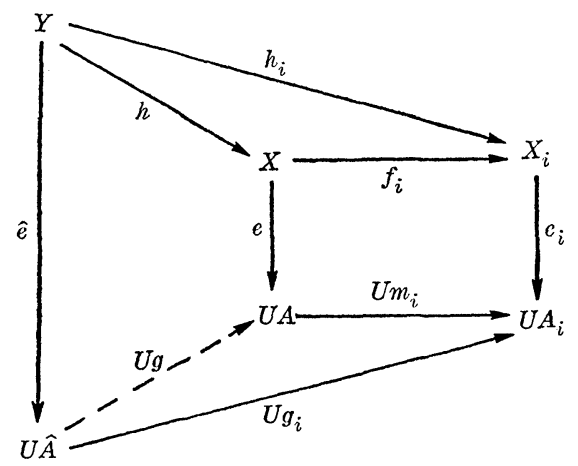

Consequently $(h, g): \hat{B} \rightarrow B$ is a $\underline{B}$-morphism with $V(h, g)=h$.

(iv) $E:(\underline{A}, U) \rightarrow(\underline{B}, V)$ preserves initiality.

Let $\left(A \stackrel{m_{i}}{\rightarrow} A_{i}\right)_{I}$ be a $U$-initial source in $\underline{A}$, let $B=(X \stackrel{e}{\rightarrow} U \widetilde{A})$ be a $\underline{B}$-object, $h: V B \rightarrow V(E A)$ be an $\underline{X}$-morphism, and $\left(B \stackrel{\left(h_{i}, g_{i}\right)}{\longrightarrow} E A_{i}\right)_{I}$ be a source in $B$ with $V\left(h_{i}, g_{i}\right)=V\left(E m_{i}\right) \cdot h$ for each $i \in I$. Since $X \stackrel{e}{\rightarrow} U \widetilde{A}$ is semi-universal and $\left(A \stackrel{m_{i}}{\rightarrow} A_{i}\right)_{I}$ is $U$-initial, there exists a unique $\underline{A}$-morphism $g: \widetilde{A} \rightarrow A$ such that the following diagram commutes:

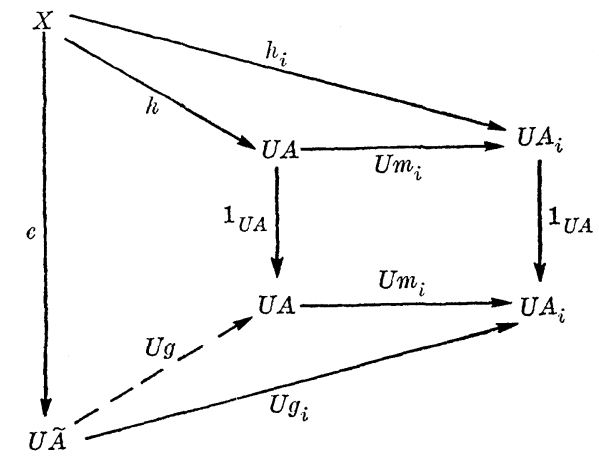

Consequently $(h, g): B \rightarrow E A$ is a $\underline{B}$-morphism with $V(h, g)=h$.

(v) $E$ is initially dense.

Let $B=(X \stackrel{e}{\rightarrow} U A)$ be any $\underline{B}$-object. Then $B \stackrel{\left(e, 1_{A}\right)}{\longrightarrow} E A$ is a $V$ initial source with domain $B$.

(vi) $E$ is universal.

Suppose that $(\underline{A}, U) \stackrel{F}{\rightarrow}(\underline{C}, W)$ is an initiality preserving functor over $\underline{X}$ with $(\underline{C}, W)$ being initially complete. Define a functor $G$ : $\underline{B} \rightarrow \underline{C}$ as follows: Any $\underline{B}$-object $B=(X \stackrel{e}{\rightarrow} U A)$ can be interpreted as a $W$-morphism $X \stackrel{e}{\rightarrow} W(F A)$. Since $(\underline{C}, W)$ is initially complete 
there is a $\underline{C}$-object (denoted by) $G B$ and a $W$-initial $\underline{C}$-morphism $G B \stackrel{e_{B}}{\rightarrow} F A$ such that

$$
W G B \stackrel{W e_{B}}{\longrightarrow} W F A=X \stackrel{e}{\longrightarrow} U A .
$$

By amnesticity of $W$, the object $G B$ and hence $e_{B}$ are uniquely determined. If $g=(f, k): B \rightarrow B^{\prime}$ is a $B$-morphism with $B^{\prime}=$ $\left(X^{\prime} \stackrel{e^{\prime}}{\rightarrow} U A^{\prime}\right)$, the diagram

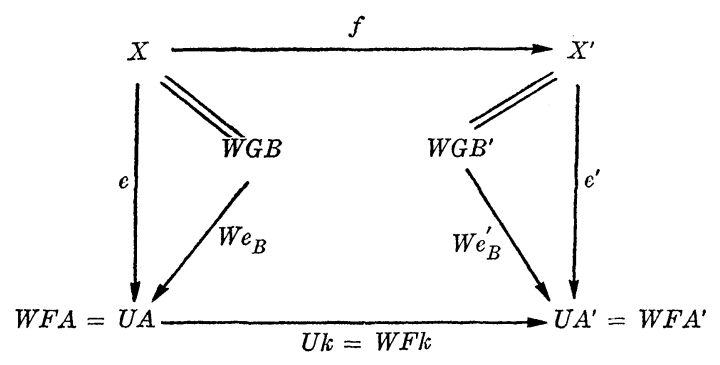

commutes. Since $G B^{\prime} \stackrel{e_{B^{\prime}}}{\rightarrow} F A^{\prime}$ is $W$-initial and $\left(W e_{B^{\prime}}\right) \cdot f=W\left(F k \cdot e_{B}\right)$ there exists a unique $\underline{C}$-morphism (denoted by) $G g: G B \rightarrow G B^{\prime}$ with $W G g=f$. The correspondence $B \stackrel{g}{\rightarrow} B^{\prime} \mapsto G B \stackrel{G_{g}}{\rightarrow} G B^{\prime}$ defines a functor $G:(\underline{B}, V) \rightarrow(\underline{C}, W)$ over $\underline{X}$. For any $\underline{A}$-object $A$ we have trivially $G E A=F A$. Since $G, E$, and $F$ are functors over $X$, this implies that $G \cdot E=F$, i.e., that $G$ is an extension of $F$. To see that $G$ preserves initiality, let $\left(B \stackrel{p_{i}}{\rightarrow} B_{i}\right)_{I}$ be a $V$-initial source in $\underline{B}$ (where $B=(X \stackrel{e}{\rightarrow} U A), \quad B_{i}=\left(X_{i} \stackrel{e_{i}}{\rightarrow} U A_{i}\right)$, and $p_{i}=\left(f_{i}, k_{i}\right)$ for each $\left.i \in I\right)$. Then by construction (see part (iii) above)

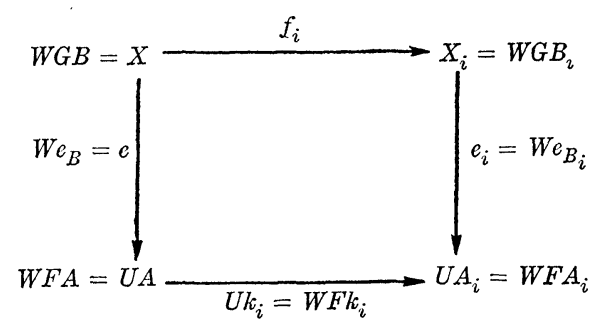

is the (semi-universal, $U$-initial)-factorization of $\left(X \stackrel{e_{i} f_{i}}{\longrightarrow} U A_{i}\right)_{I}$. To see that $\left(G B \stackrel{G p_{i}}{\longrightarrow} G B_{i}\right)_{I}$ is $W$-initial let $h: W C \rightarrow W G B$ be an $\underline{X}$-morphism and $\left(C \stackrel{g_{i}}{\rightarrow} G B_{i}\right)$ be a source in $\underline{C}$ with $\left(W G p_{i}\right) \cdot h=W g_{i}$ for each $i \in I$. Since $\left(A \stackrel{k_{i}}{\rightarrow} A_{i}\right)_{I}$ is $U$-initial and $F$ preserves initiality, the $\underline{C}$-source $\left(F A \stackrel{F k_{i}}{\longrightarrow} F A_{i}\right)_{I}$ is $W$-initial. Hence $W\left(F k_{i}\right) \cdot e \cdot h=$ $W\left(e_{B_{i}} \cdot g_{i}\right)$ implies that there exists a $\underline{C}$-morphism $g: C \rightarrow F A$ with 
$W g=e \cdot h$. Since $G B \stackrel{e_{B}}{\rightarrow} F A$ is $W$-initial by construction of $G$, and $\left(W e_{B}\right) \cdot h=W g$, there exist a $\underline{C}$-morphism $\bar{h}: C \rightarrow G B$ with $W \bar{h}=h$.

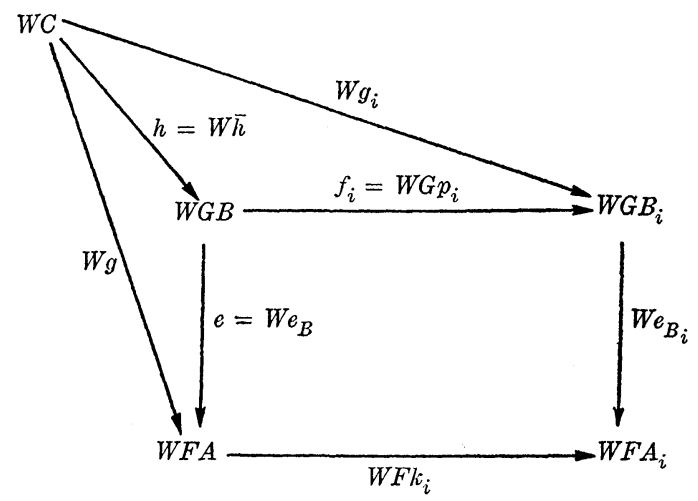

Consequently $\left(G B \stackrel{G p_{i}}{\longrightarrow} G B_{i}\right)_{I}$ is $W$-initial. Since $E$ is initially dense, $G:(\underline{B}, V) \rightarrow(\underline{C}, W)$ is the only initiality preserving extension of $F$. This completes the proof.

Surprisingly enough, the property of the above proposition actually characterizes topologically-algebraic functors, since we have:

Proposition 2.6. If the category over $\underline{X},(\underline{A}, U)$, has a reflective universal initial completion, then $U: \underline{A} \rightarrow \underline{X}$ is topologicallyalgebraic.

Proof. Let $(\underline{A}, U) \stackrel{E}{\hookrightarrow}(\underline{B}, V)$ be a reflective universal initial completion of $(A, U)$. For simplicity, let us assume that $(\underline{A}, U)$ is a full, reflective subcategory of $\underline{B}$, and $E$ is the corresponding embedding. To show that $U: \underline{A} \rightarrow \underline{X}$ is topologically-algebraic let $\mathscr{S}=\left(X \stackrel{f_{i}}{\longrightarrow} U A_{i}\right)_{I}$ be any $U$-source. We must show that it has a (generating, $U$-initial)-factorization. Let $\hat{\mathscr{S}}=\left(X \stackrel{f_{j}}{\rightarrow} U A_{j}\right)_{J}$ be the standard enrichment of $\mathscr{S}(1.6)$. Since $(B, V)$ is initially complete, there exists a $V$-initial source $\left(B \stackrel{\overline{f_{j}}}{\rightarrow} A_{j}\right)_{J}$ such that $V B=X$ and $V \bar{f}_{j}=f_{j}$ for each $j \in J$. Let $e: B \rightarrow A$ be the $\underline{A}$-reflection map for $B$. Then for each $j \in J$ there exists a unique $\underline{A}$-morphism $m_{j}: A \rightarrow$ $A_{j}$ with $m_{j} \cdot e=\bar{f}_{j}$. Applying $V$, we obtain a factorization $X \stackrel{f_{j}}{\rightarrow} U A_{j}=X \stackrel{V e}{\rightarrow} U A \stackrel{U m_{j}}{\longrightarrow} U A_{j}$ of $\hat{\mathscr{S}}$. Clearly $X \stackrel{V e}{\rightarrow} U A$ is a generating $U$-morphism. By Proposition 1.7 it remains to show that $X \stackrel{V e}{\rightarrow} U A$ belongs to $\hat{\mathscr{S}}$.

To this end, construct the following category, $(\underline{C}, W)$, over $\underline{X}$ : $\underline{C}$-objects are all pairs $(H, B)$ with $B$ a $\underline{B}$-object and $H$ a set of 
$V$-morphisms $X \stackrel{h}{\rightarrow} V B$ with domain $X$ and codomain $B$. For each $\underline{C}$-object $(H, B)$ denote by $S(H, B)$ the set of all $\underline{B}$-morphisms $g: B \rightarrow$ $A$ with domain $B$, codomain in $\underline{A}$, and such that for each $h \in H$ the $U$-morphism $X \stackrel{h}{\rightarrow} V B \stackrel{V g}{\rightarrow} U A$ belongs to $\hat{\mathscr{S}} \cdot \underline{C}$-morphisms $f:(H, B) \rightarrow$ $\left(H^{\prime}, B^{\prime}\right)$ are all $\underline{B}$-morphisms $f: B \rightarrow B^{\prime}$ with the property that $g \in$ $S\left(H^{\prime}, B^{\prime}\right)$ implies $g f \in S(H, B)$ [equivalently: such that $B^{\prime} \stackrel{g}{\rightarrow} A$ in $S\left(H^{\prime}, B^{\prime}\right)$, and $X \stackrel{h}{\rightarrow} V B$ in $H$, implies $X \stackrel{h}{\rightarrow} V B \stackrel{V f}{\rightarrow} V B^{\prime} \stackrel{V g}{\longrightarrow} U A$ is in $\hat{\mathscr{P}]}$. $V f$.

$W: \underline{C} \rightarrow \underline{X}$ is defined by $W(H, B)=V B$ and $W\left((H, B) \stackrel{f}{\rightarrow}\left(H^{\prime}, B^{\prime}\right)\right)=$

$(\underline{C}, W)$ is a category over $\underline{X}$. (The fact, that $W$ usually fails to be amnestic, is irrelevant.) $(\underline{C}, W)$ is initially complete. To see this, let $\left(Y \stackrel{r_{k}}{\rightarrow} W\left(H_{k}, B_{k}\right)\right)_{K}$ be a $W$-source. Since $(\underline{B}, V)$ is initially complete, there exists a $V$-initial source $\left(B \stackrel{\bar{r}_{k}}{\rightarrow} B_{k}\right)_{K}$ with $V\left(B \stackrel{\vec{r}_{k}}{\rightarrow} B_{k}\right)_{K}=$ $\left(Y \stackrel{r_{k}}{\rightarrow} V B_{k}\right)_{K}$. Let $H$ be the set of all $V$-morphisms $X \stackrel{h}{\rightarrow} V B$ with the property that for each $k \in K$ and each $g \in S\left(H_{k}, B_{k}\right)$ the $U$-morphism $X \stackrel{h}{\rightarrow} V B \stackrel{r_{k}}{\rightarrow} V B_{k} \stackrel{V g}{\longrightarrow} U A$ belongs to $\hat{\mathscr{\rho}}$. Then $\left((H, B) \stackrel{\bar{r}_{k}}{\rightarrow}\right.$ $\left.\left(H_{k}, B_{k}\right)\right)_{K}$ is easily seen to be $W$-initial. Next, define $F:(\underline{A}, U) \rightarrow$ $(\underline{C}, W)$ by

$$
F\left(A \stackrel{f}{\longrightarrow} A^{\prime}\right)=(\phi, A) \stackrel{f}{\longrightarrow}\left(\phi, A^{\prime}\right) .
$$

$F$ preserves initiality. To see this, let $\left(A \stackrel{n_{k}}{\rightarrow} A_{k}\right)_{K}$ be a $U$-initial source, let $g: W(H, B) \rightarrow W F A$ be a $\underline{X}$-morphism, and let $\left((H, B) \stackrel{g_{k}}{\rightarrow}\right.$ $\left.F A_{k}\right)_{K}$ be a source in $\underline{C}$ with $\left(W F n_{k}\right) \cdot g=W g_{k}$ for each $k \in K$. Since $E:(\underline{A}, U) \rightarrow(\underline{B}, V)$ preserves initiality, $\left(A \stackrel{n_{k}}{\rightarrow} A_{k}\right)_{K}$ is $V$-initial. Hence $\left(V n_{k}\right) \cdot g=V g_{k}$ implies that there exists a $\underline{B}$-morphism $\bar{g}: B \rightarrow F A$ with $V \bar{g}=g$. To show that $(H, B) \stackrel{\bar{g}}{\rightarrow} F A$ is a $\underline{C}$-morphism,

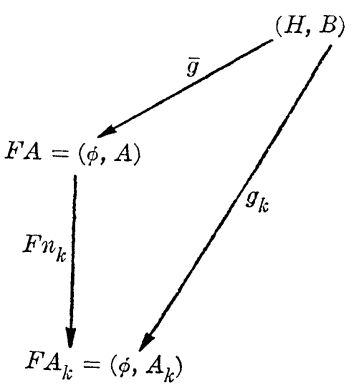

let $X \stackrel{h}{\rightarrow} V B$ be an element of $H$. Since all $g_{k}$ are $\underline{C}$-morphisms and $1_{A_{k}} \in S\left(\phi, A_{k}\right)$, this implies that all $X \stackrel{h}{\rightarrow} V B \stackrel{g}{\rightarrow} U A \stackrel{U n_{k}}{\longrightarrow} U A_{k}=$ $X \stackrel{h}{\rightarrow} V B \stackrel{V g_{k}}{\longrightarrow} U A_{k}$ belong to $\hat{\mathscr{S}}$. Since $\left(A \stackrel{n_{k}}{\rightarrow} A_{k}\right)_{K}$ is $U$-initial, 1.6(c) 
implies that $X \stackrel{h}{\rightarrow} V B \stackrel{g}{\rightarrow} U A$ belongs to $\hat{\mathscr{S}}$. Thus $\bar{g} \in S(H, B)$, which implies by $1.6(\mathrm{~b})$ that $(H, B) \stackrel{\bar{g}}{\rightarrow} F A$ is a $\underline{C}$-morphism with $W \bar{g}=g$.

Since $(B, V)$ is a universal initial completion there exists an initiality preserving extension $(\underline{B}, V) \stackrel{G}{\rightarrow}(\underline{C}, W)$ of $F$ over $\underline{X}$. Recall that $\left(B \stackrel{f_{j}}{\longrightarrow} E A_{j}\right)_{J}$ is $V$-initial. Thus $\left(G B \stackrel{G f_{j}}{\longrightarrow} G A_{j}\right)_{J}=\left((H, B) \stackrel{G f_{j}}{\longrightarrow}\right.$ $\left.\left(\phi, A_{j}\right)\right)_{J}$ is $W$-initial where, by the above contruction, $H$ is the set of all $V$-morphisms $X \stackrel{h}{\rightarrow} V B$ such that $X \stackrel{h}{\rightarrow} V B \stackrel{f_{i}}{\rightarrow} U A_{j}$ belongs to $\hat{\mathscr{S}}$. In particular $X \stackrel{1_{x}}{\rightarrow} V B$ belongs to $H$. However, $B \stackrel{e}{\rightarrow} E A$ is a $\underline{B}$-morphism, so that $G B \stackrel{G e}{\rightarrow} F A$ is a $\underline{C}$-morphism; i.e., $(H, B) \stackrel{e}{\rightarrow}(\phi, A)$ is a $C$-morphism, so that for each $h \in H$, eh must belong to $\hat{\mathscr{S}}$. In particular $e=e \cdot 1_{X}$ must belong to $\hat{\mathscr{S}}$, which is what was to be shown. Hence $(A, U)$ is topologically-algebraic.

Putting the last two propositions together yield ${ }_{\imath}$ our main result.

THEOREM 2.7. The following are equivalent:

(1) $U$ is topologically-algebraic.

(2) $(\underline{A}, U)$ has a reflective universal initial completion.

THEOREM 2.8. The following are equivalent:

(1) $U$ is semi-topological.

(2) $(\underline{A}, U)$ has a reflective Mac Neille completion.

(3) $U$ is the full reflective restriction of some topological functor.

(4) $U$ belongs to the smallest class of functors that contains all topologically-algebraic functors and is closed under composition.

Proof. $(1) \Rightarrow(2)$. The proof of this is similar to that of Proposition 2.5 where objects of the completion are all semi-final $U$ morphisms.

$(2) \Rightarrow(3)$. Trivial.

$(3) \Rightarrow(4)$. Full reflective embeddings and topological functors are topologically-algebraic.

$(4) \Rightarrow(1)$. Every topologically-algebraic functor is semi-topological and semi-topological functors are closed under composition.

It should be noted that the equivalence of (1) and (3) above has been obtained previously be Tholen and Wischnewsky [24] and the equivalence of (1) and (2) by Hoffmann [13]. 
3. Examples. Since the initial completions of $\S 2$ are obtained constructively, it is of interest to see what the construction yields in some special cases:

Topological examples: Let $\underline{A}$ be any epireflective subcategory of Top, consisting of $T_{0}$-spaces only and containing at least one space with more than one element. Let $U: A \rightarrow$ Set be the usual forgetful functor.

Proposition 3.1. For any U-morphism $X \stackrel{e}{\rightarrow} U A$ the following are equivalent:

(1) $X \stackrel{e}{\rightarrow} U A$ is semi-final.

(2) $X \stackrel{e}{\rightarrow} U A$ is semi-universal.

(3) $X \stackrel{e}{\rightarrow} U A$ is surjective.

Proof. $\quad(1) \Rightarrow(2)$. Proposition 1.5.

$(2) \Longrightarrow(3)$. Let $X \stackrel{e}{\rightarrow} U A=X \stackrel{g}{\rightarrow} U B \stackrel{U m}{\longrightarrow} U A$ be the (surjective, embedding)-factorization in Top. Then $B$ belongs to $A$, since $A$ is epireflective in Top, and $B \stackrel{m}{\rightarrow} A$ is $U$-initial. Hence there exists a unique $A$-morphism $f: A \rightarrow B$ such that the diagram

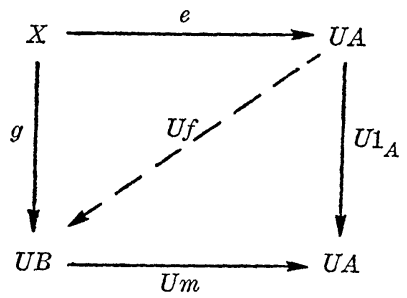

commutes. So $U m$ is a retraction, hence surjective. Consequently $e=(U m) \cdot g$ is surjective.

$(3) \Rightarrow(1)$. If $X \stackrel{e}{\rightarrow} U A$ is surjective let $f: U A \rightarrow X$ be any function such that $e f=1_{U A}$. Then $X \stackrel{e}{\rightarrow} U A$ is the semi-final solution of the $U$-sink consisting of the $U$-comorphism $f$ together with all $U$-comorphisms $U A_{i} \stackrel{f_{i}}{\rightarrow} X$ having the property that $e f_{i}$ is a constant function.

3.2. As a consequence of the above, for any such category $(\underline{A}, U)$ over Set the universal initial completion agrees with the Mac Neille completion and the objects of either are obtained by "blowing up points" in the spaces belonging to $\underline{A}$. Thus we have 


\begin{tabular}{|c|c|}
\hline Category $A$ & $\begin{array}{l}\text { Universal initial completion }= \\
\text { Mac Neille completion }\end{array}$ \\
\hline all $T_{0}$-spaces & all topological spaces \\
\hline all $T_{1}$-spaces & $\begin{array}{l}\text { all symmetric topological }\left(=R_{0}^{-}\right) \\
\text {spaces }\end{array}$ \\
\hline all $T_{2}$-spaces & all $H O$-spaces of $\mathrm{K}$. Császár \\
\hline $\begin{array}{c}\text { all } T_{3} \text {-spaces } \\
\left(=\text { regular }+T_{1} \text {-spaces }\right)\end{array}$ & all regular spaces \\
\hline $\begin{array}{l}\text { all Tychonoff spaces } \\
\text { (= completely regular }+T_{1^{-}} \\
\text {spaces })\end{array}$ & all completely regular spaces \\
\hline all zero dimensional $T_{0}$-spaces & all zero-dimensional spaces \\
\hline
\end{tabular}

3.3. Analogously, the universal initial completion (resp. Mac Neille completion) of the concrete category of separated uniform spaces is the concrete category of all uniform spaces.

\section{Algebraic examples:}

Proposition 3.4. Let $U: \underline{A} \rightarrow \underline{X}$ be a regular functor in the sense of [4] and suppose that every U-initial source is a monosource. Then the following are equivalent:

(1) $X \stackrel{e}{\rightarrow} U A$ is semi-universal.

(2) $X \stackrel{e}{\rightarrow} U A$ extremally generates $A$; i.e., it generates $A$ in the usual algebraic sense.

Proof.

$(1) \Rightarrow(2)$. Let $X \stackrel{e}{\rightarrow} U A=X \stackrel{g}{\rightarrow} U B \stackrel{U m}{\longrightarrow} U A$ be an (extremally generating, mono)-factorization. Then $B \stackrel{m}{\rightarrow} A$ is initial. Hence there exists a unique $\underline{A}$-morphism $f: A \rightarrow B$ such that the diagram

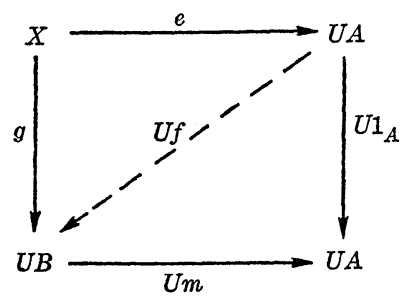

commutes. Consequently $m$ is an isomorphism, i.e., $X \stackrel{e}{\rightarrow} U A$ extrem- 
ally generates $A$.

$(2) \Longrightarrow(1)$. Let $\left(B \stackrel{m_{2}}{\rightarrow} A_{i}\right)_{I}$ be an initial source, $\left(A \stackrel{f_{i}}{\rightarrow} A_{i}\right)_{I}$ be an arbitrary source, and $X \stackrel{f}{\rightarrow} U B$ a $U$-morphism with $U m_{i} \cdot f=U f_{i} \cdot e$ for each $i \in I$. Let

$$
X \stackrel{e}{\longrightarrow} U A=X \stackrel{\eta_{X}}{\longrightarrow} U F X \stackrel{U \bar{e}}{\longrightarrow} U A
$$

and

$$
X \stackrel{\vec{f}}{\longrightarrow} U B=X \stackrel{\eta_{X}}{\longrightarrow} U F X \stackrel{U f}{\longrightarrow} U B
$$

be (universal, -)-factorizations, where $F$ denotes the left-adjoint of $U$. Then $\bar{e}$ is a regular epimorphism. Since $\left(B \stackrel{m_{i}}{\rightarrow} A_{\imath}\right)$ is a monosource there exists a unique $A$-morphism $g: A \rightarrow B$ such that the diagram

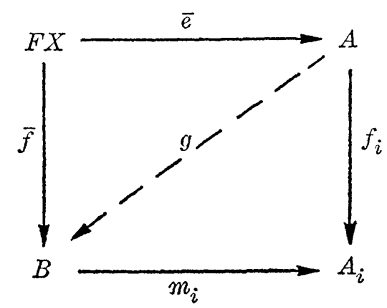

commutes. Hence $g: A \rightarrow B$ is the unique morphism making the diagram

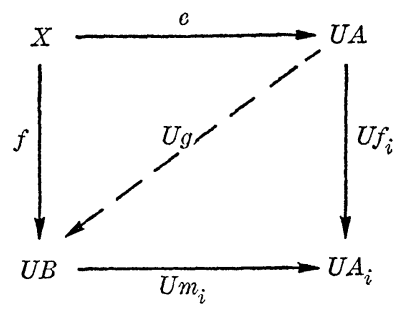

commute.

For the following categories the forgetful functors to Set satisfy the above conditions:

R-Mod, Grp, Lat, Comp Haus.

In general for these categories the universal initial completions are much larger than the Mac Neille completions; e.g.,

$$
\begin{aligned}
\text { Comp Haus } & \varsubsetneqq \begin{array}{c}
\text { Mac Neille completion } \\
\text { of Comp Haus }
\end{array} \\
& \varsubsetneqq \text { Universal initial completion of Comp Haus } .
\end{aligned}
$$


The universal initial completion of Comp Haus is the category Prox of all (not necessarily $T_{1}$ ) proximity spaces and proximal mapsequivalently, the category of totally bounded uniform spaces and uniformly continuous maps. Analogously, the universal initial completion of the concrete category of separated complete uniform spaces is the concrete category of all uniform spaces. A corresponding result holds for regular nearness spaces.

3.5. In general, for regular categories, initial sources need not be mono-sources; e.g.:

(1) for the concrete category of sets, every source is initial;

(2) for the concrete category of pointed sets, the following are equivalent:

(i ) $\left((X, p) \stackrel{m_{2}}{\rightarrow}\left(X_{i}, p_{i}\right)\right)_{I}$ is initial.

(ii) if $m_{i}(x)=p_{i}$ for each $i \in I$, then $x=p$.

In the last case the universal initial completion coincides with the Mac Neille completion and is the category of all pairs of sets $(X, S)$ with $S \subset X$ and all functions preserving the distinguished subsets.

3.6. Let Grph be the concrete category of graphs. Objects are triples $(X, d, c)$ where $X$ is a set and $d$ and $c$ are unary operations on $X$, satisfying:

$$
c d=d^{2}=d \text { and } d c=c^{2}=c .
$$

The elements of $c[X]=d[X]$ are called vertices and the elements of $X \backslash c[X]$ are called edges. Morphisms are functions commuting with the operations $c$ and $d$.

(a) A source $\left((X, d, c) \stackrel{f_{i}}{\rightarrow}\left(X_{i}, d_{i}, c_{i}\right)\right)_{I}$ in Grph is initial if and only if: (for each $i \in I, f_{i} x=f_{i} y$ is a vertex) $\Rightarrow(x=y$ is a vertex).

(b) $Y \stackrel{e}{\rightarrow}(X, d, c)$ is generating if and only if $e[Y]$ contains all edges of $(X, d, c)$.

(c) $Y \stackrel{e}{\rightarrow}(X, d, c)$ is semi-universal if and only if each edge in $(X, d, c)$ has precisely one preimage under $e$.

(d) $Y \stackrel{e}{\rightarrow}(X, d, c)$ is semi-final if and only is it is semi-universal and it satisfies:

$$
e^{-1}(c x)=\phi \Longleftrightarrow e^{-1}(d x)=\phi .
$$

Hence the Mac Neille completion of Grph is properly contained in the universal initial completion of Grph. The objects of the latter are obtained essentially by replacing the set of vertices in a graph by a family of pairwise disjoint (possibly empty) sets. Grph is 
simultaneously reflective and mono-coreflective (but not bi-coreflective) in its universal initial completion.

\section{Factorizations:}

3.7. Theorem 2.1 implies that for every $(\underline{E}, \underline{M})$-category $\underline{X}, \underline{E}$ must be contained in the class of all epimorphisms of $\underline{X}$. In particular, this implies that the category Set has precisely two factorization structures, namely:

(1) the trivial one; i.e., the (iso, source)-factorization structure given by the (bijective, source)-factorizations,

(2) the regular one; i.e., the (regular epi, mono-source)-factorization structure, given by the (surjective, point-separating source)factorization.

3.8. None of the implications:

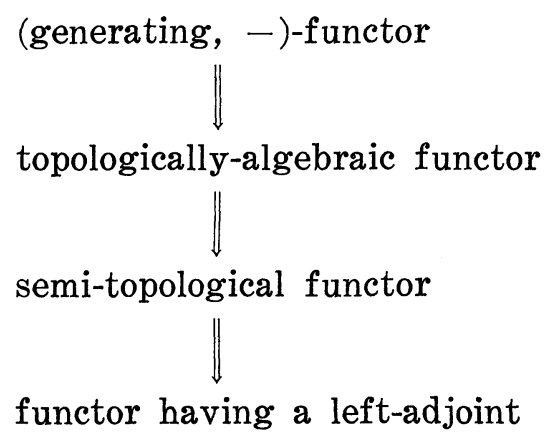

is reversible. For the first consider the identity functor on any category that is not an (epi, -)-category; for the second see [7]; for the third consider the functor from a noncomplete partially ordered class with least element (regarded as a category in the usual way) into the terminal category. However, in the case that $\underline{A}$ is an (epi, -)-category, all of the above conditions are equivalent.

\section{REFERENCES}

1. J. Adámek, Colimits of algebras revisited, Bull. Austral. Math. Soc., 17 (1977), 433-450.

2. J. Adámek, H. Herrlich and G. E. Strecker, Least and largest initial completions I and II, Comment, Math. Univ. Carolinae, 20 (1979), 43-77.

3. G. C. L. Brümmer, Struktuurfunktore en faktorisering, Proc. S. Afr. Math. Soc., 4 (1974), 81-83.

4. H. Herrlich, Topological functors, General Topol. Appl., 4 (1974), 125-142.

5. — Regular categories and regular functors, Canad. J. Math., 26 (1974), 709-720.

6. —, Initial completions, Math. Zeitschr., 150 (1976), 101-110. 
7. H. Herrich, R. Nakagawa, G. E. Strecker, and T. Titcomb, Equivalence of topologically-algebraic and semi-topological functors. Canad. J. Math., to appear

8. R.-E. Hoffman, $(E, M)$-universally topological functors, Habilitationsschrift, Düsseldorf, 1974.

9. Semi-identifying lifts and a generalization of the duality theorem for topological functors, Math. Nachr., 74 (1976), 295-307.

10. - Topological functors admitting generalized Cauchy-completions, Springer Lecture Notes Math., 540 (1976), 286-344.

11. - Topological completion of faithful functors, Kategorienseminar 1, Hagen (1976), 26-37.

12. - Full reflective restrictions of topological functors, Math. Coll. Univ. Cape Town, 11 (1977), 65-88.

13. W Note on semi-topological functors, Math. Z., 160 (1978), 69-74.

14. S. S. Hong, Categories in which every mono-source is initial, Kyungpook Math. J., 15 (1975), 133-139.

15. Y. H. Hong, Studies on categories of universal topological algebras, Thesis, McMaster University, 1974.

16. M. Hušek, S-Categories, Comment. Math. Univ. Carolinae, 5 (1964), 37-46.

17. L. Kučera and A. Pultr, On a mechanism of defining morphisms in concrete categories, Cahiers Topol. Geom. Diff., 13 (1972), 397-410.

18. Th. Marny, Rechts-Bikategoriestrukturen in topologischen Kategorien Thesis, F. U. Berlin, 1973.

19. W. Tholen, On Wyler's taut lift theorem, General Topol. Appl., 8 (1978), 197-206.

20. —_, Zum Satz von Freyd und Kelly, Math. Ann., 232 (1978), 1-14.

21. — $M$-functors, Nordwestdeutsches Kategorienseminar, Mathematik-Arbeitspapiere, 7 (Bremen 1976), 178-185.

22. - Semi-topological functors and adjoint liftings, Kategorientagung Oberwolfach, 1977.

23. — Semi-topological functors I, J. Pure Applied Algebra, 15 (1979), 53-73.

24. W. Tholen and M. B. Wischnewsky, Semi-topological functors II: External characterization J. Pure Applied Algebra 15 (1979), 75-92.

25. V. Trnková, Automata and categories, Lecture Notes Computer Sci., 32 (1975), 138-152.

26. M. B. Wischnewsky, A lifting theorem for right adjoints, Cahiers Topol. Geom. Diff., 19 (1978), 155-168.

27. H. Wolff, Topological functors and right adjoints, General Topol. Appl., 9 (1978), 101-110.

28. - Topological functors and lifting adjoints, preprint.

29. ' O. Wyler, Top categories and categorical topology, General Topol. Appl., 1 (1971), 17-28.

30. On the categories of general topology and topological algebra, Archiv Math., 22 (1971), 7-17.

Received May 1, 1978.

UNIVERSITÄT BREMEN

28 BREMEN

Fed. Rep. Germany

AND

KaNSAS State UNIVERSITY

ManhatTAN, KS 66502 


\section{PACIFIC JOURNAL OF MATHEMATICS}

\section{EDITORS}

Donald BABBITT (Managing Editor)

University of California

Los Angeles, CA 90024

HUGo RossI

University of Utah

Salt Lake City, UT 84112

C. C. MOORE and ANDREW OGG

University of California

Berkeley, CA 94720

\section{J. DuGUNDJI}

Department of Mathematics

University of Southern California

Los Angeles, CA 90007

R. FINN and J. Milgram

Stanford University

Stanford, CA 94305

ASSOCIATE EDITORS
E. F. BECKENBACH
B. H. NeumanN
F. WOLF
K. YoSHIDA

\section{SUPPORTING INSTITUTIONS}

\author{
UNIVERSITY OF BRITISH COLUMBIA \\ CALIFORNIA INSTITUTE OF TECHNOLOGY \\ UNIVERSITY OF CALIFORNIA \\ MONTANA STATE UNIVERSITY \\ UNIVERSITY OF NEVADA, RENO \\ NEW MEXICO STATE UNIVERSITY \\ OREGON STATE UNIVERSITY \\ UNIVERSITY OF OREGON
}

\author{
UNIVERSITY OF SOUTHERN CALIFORNIA \\ STANFORD UNIVERSITY \\ UNIVERSITY OF HAWAII \\ UNIVERSITY OF TOKYO \\ UNIVERSITY OF UTAH \\ WASHINGTON STATE UNIVERSITY \\ UNIVERSITY OF WASHINGTON
}

The Supporting Institutions listed above contribute to the cost of publication of this Journal, but they are not owners or publishers and have no responsibility for its content or policies.

Mathematical papers intended for publication in the Pacific Journal of Mathematics should be in typed form or offset-reproduced, (not dittoed), double spaced with large margins. Please do not use built up fractions in the text of the manuscript. However, you may use them in the displayed equations. Underline Greek letters in red, German in green, and script in blue. The first paragraph or two must be capable of being used separately as a synopsis of the entire paper. Please propose a heading for the odd numbered pages of less than 35 characters. Manuscripts, in triplicate, may be sent to any one of the editors. Please classify according to the scheme of Math. Reviews, Index to Vol. 39. Supply name and address of author to whom proofs should be sent. All other communications should be addressed to the managing editor, or Elaine Barth, University of California, Los Angeles, California, 90024.

50 reprints to each author are provided free for each article, only if page charges have been substantially paid. Additional copies may be obtained at cost in multiples of 50 .

The Pacific Journal of Mathematics is issued monthly as of January 1966. Regular subscription rate: $\$ 84.00$ a year (6 Vols., 12 issues). Special rate: $\$ 42.00$ a year to individual members of supporting institutions.

Subscriptions, orders for numbers issued in the last three calendar years, and changes of address should be sent to Pacific Journal of Mathematics, P.O. Box 969, Carmel Valley, CA 93924, U.S.A. Older back numbers obtainable from Kraus Periodicals Co., Route 100, Millwood, NY 10546.

PUBLISHED BY PACIFIC JOURNAL OF MATHEMATICS, A NON-PROFIT CORPORATION

Printed at Kokusai Bunken Insatsusha (International Academic Printing Co., Ltd.). 8-8, 3-chome, Takadanobaba, Shinjuku-ku, Tokyo 160, Japan.

Copyright (C) 1979 by Pacific Journal of Mathematics Manufactured and first issued in Japan 


\section{Pacific Journal of Mathematics}

\section{Vol. 82 , No. 2 \\ February, 1979}

Krishnaswami Alladi and Paul Erdős, On the asymptotic behavior of large prime

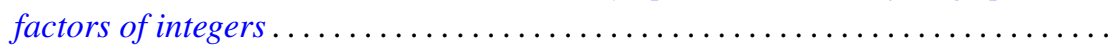

Alfred David Andrew, A remark on generalized Haar systems in $L_{p}$,

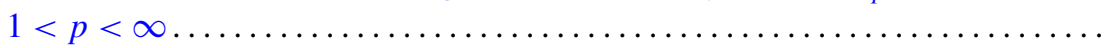

John M. Baker, A note on compact operators which attain their norm . . ........

Jonathan Borwein, Weak local supportability and applications to

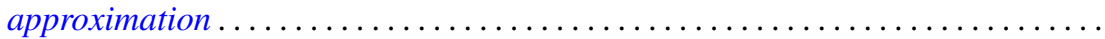

Tae Ho Choe and Young Soo Park, Wallman's type order compactification ........

Susanne Dierolf and Ulrich Schwanengel, Examples of locally compact

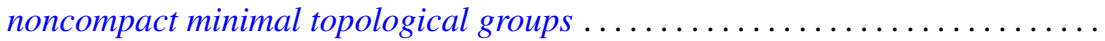

Michael Freedman, A converse to (Milnor-Kervaire theorem) $\times R$ etc. . . . . . . .

George Golightly, Graph-dense linear transformations ..................

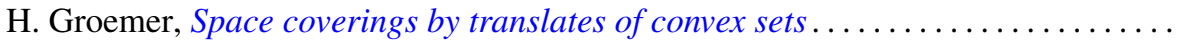

Rolf Wim Henrichs, Weak Frobenius reciprocity and compactness conditions in

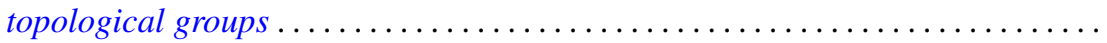

Horst Herrlich and George Edison Strecker, Semi-universal maps and universal

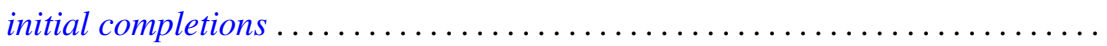

Sigmund Nyrop Hudson, On the topology and geometry of arcwise connected,

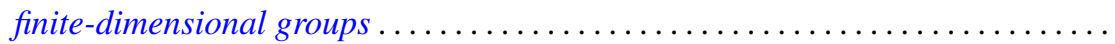

K. John and Václav E. Zizler, On extension of rotund norms. II .............

Russell Allan Johnson, Existence of a strong lifting commuting group of transformations. II.

Bjarni Jónsson and Ivan Rival, Lattice varieties covering the smallest nonmodular variety

Grigori Abramovich Kolesnik, On the order of Dirichlet L-functions .

Robert Allen Liebler and Jay Edward Yellen, In search of nonsolvable groups of

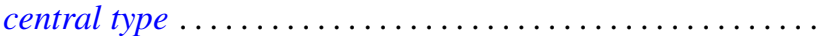

Wilfrido Martínez T. and Adalberto Garcia-Maynez Cervantes, Unicoherent plane Peano sets are $\sigma$-unicoherent ...

M. A. McKiernan, General Pexider equations. I. Existence of injective

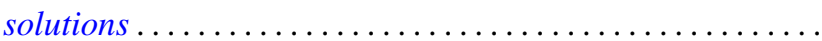

M. A. McKiernan, General Pexider equations. II. An application of the theory of webs.

Jan K. Pachl, Measures as functionals on uniformly continuous functions . .

Lee Albert Rubel, Convolution cut-down in some radical convolution algebras ...

Peter John Slater and William Yslas Vélez, Permutations of the positive integers

with restrictions on the sequence of differences. II . . .

Raymond Earl Smithson, A common fixed point theorem for nested spaces ....

Indulata Sukla, Generalization of a theorem of McFadden .... . . .

Jun-ichi Tanaka, A certain class of total variation measures of analytic measures.

Kalathoor Varadarajan, Modules with supplements .............. 